\title{
Human hand radiography using X-ray differential phase contrast combined with dark-field imaging
}

\section{Journal Article}

\section{Author(s):}

Thüring, Thomas; Guggenberger, Roman; Alkadhi, Hatem; Hodler, Jürg; Vich, Magdalena; Wang, Zhentian; David, Christian; Stampanoni, Marco

Publication date:

2013-06

\section{Permanent link:}

https://doi.org/10.3929/ethz-b-000066167

\section{Rights / license:}

In Copyright - Non-Commercial Use Permitted

Originally published in:

Skeletal Radiology 42(6), https://doi.org/10.1007/s00256-013-1606-7 


\title{
Human hand radiography using X-ray differential phase contrast combined with dark-field imaging
}

\author{
Thomas Thüring • Roman Guggenberger • \\ Hatem Alkadhi • Jürg Hodler • Magdalena Vich • \\ Zhentian Wang • Christian David • Marco Stampanoni
}

Received: 1 February 2013 /Revised: 7 March 2013 / Accepted: 18 March 2013 /Published online: 7 April 2013

(C) ISS 2013

\section{Introduction}

Established X-ray-based imaging procedures such as conventional radiography and computed tomography (CT) rely on the interaction of photons when passing through tissue, including the Compton scattering and the photoelectric effect, which is influenced by the X-ray energy and the type of matter. The resulting mean attenuation of X-rays can be measured and depicted on images with different gray levels.

$\mathrm{X}$-ray phase contrast imaging $(\mathrm{PCI})$ represents a relatively new imaging technique relying upon the refraction of $\mathrm{X}$ rays. As such, PCI relies on a fundamentally different physical contrast mechanism compared with conventional, absorption-based X-ray imaging. In the energy range of

Electronic supplementary material The online version of this article (doi:10.1007/s00256-013-1606-7) contains supplementary material, which is available to authorized users

T. Thüring $(\bowtie) \cdot$ Z. Wang $\cdot$ C. David $\cdot$ M. Stampanoni Paul Scherrer Institut, WBBA/213, 5232, Villigen, Switzerland e-mail: thomas.thuering@psi.ch

T. Thüring $\cdot$ M. Stampanoni

Institute for Biomedical Engineering,

Swiss Federal Institute of Technology,

Zurich,

Switzerland

R. Guggenberger $\cdot$ H. Alkadhi $\cdot$ J. Hodler Institute of Diagnostic and Interventional Radiology, University Hospital Zurich, Rämistrasse 100, 8091, Zurich, Switzerland

M. Vich

Institute of Anatomy, University Zurich, Winterthurerstrasse 190, 8057, Zurich, Switzerland diagnostic imaging (10-120 keV), refraction is the dominant effect over absorption, but more difficult to acquire. Previous studies have demonstrated that PCI can provide considerably higher contrast in soft tissue, giving rise to its application in fields where conventional radiography and CT are usually limited.

Among a variety of techniques used to acquire phase contrast images, grating interferometry [1] has recently attracted great attention because of its compatibility with conventional X-ray tubes $[2,3]$, which is the key prerequisite for the clinical applicability. In addition, this technique provides a third contrast mode along with absorption and phase contrast, which is the dark-field contrast [4]. Similarly, dark-field imaging again exploits a physically different interaction mechanism and represents the intensity of the scattered X-rays within the area of a single detector pixel. Image pixels with high gray values indicate strong scattering.

Recent studies have investigated the performance of phase contrast (PC) and dark-field contrast (DC) in the imaging of female breast tissue, indicating promising results for distinguishing microcalcifications and the malignant conversion or extension of the carcinoma into normal breast tissue $[5,6]$. Yet, joint pathologies such as rheumatoid arthritis, crystal arthropathies, and connective tissue diseases (e.g., scleroderma), are also associated with soft tissue affection and occasional calcifications.

Conventional radiography of the hand is a cornerstone imaging study for the detection and monitoring of joint diseases as subtle changes of joint space and bones (narrowing and erosions or osteophytes) and - if perceivable - of soft tissue (including calcifications and fibrosis) [7, 8], indicating disease activity and/or progress. While tissue evaluation with conventional radiography is based on morphological criteria 
that are reflected by changes in X-ray attenuation, PC and DC images could provide additional and/or complementary information regarding bone and soft tissue architecture and integrity.

Regarding musculoskeletal applications, experimental results using synchrotron radiation and the crystal analyzerbased technique in tomographic mode have shown structural cartilage matrix properties in human patellae samples in vitro [9]. Further, PC and DC imaging with an X-ray tube setup has been demonstrated on human finger joint radiographs (e.g., of the tendon) [10] and on infant hand tomographs [11] to study soft tissue contrast compared with the standard absorptionbased imaging.

Here, grating-based PC and DC radiography of the entire adult human hand including the wrist is demonstrated. A bidirectional acquisition scheme $[12,13]$ was applied to extend the sensitivity of the PC and DC measurements to two dimensions. For the data post-processing, a practical method for the efficient fusion of the different contrast modalities was implemented.

It is hypothesized that image information based on PC and DC imaging of the human hand in combination with the presented acquisition and image fusion methods may increase the visualization of soft tissue and/or bones compared with conventional radiography. Using an interface where the amount of fusion of the PC image can be interactively changed on-site from none to maximal, the radiologist can produce and graphically depict image information in an additional dimension. Hence, the purpose of this study was to show the feasibility and evaluate the visualization of subchondral bones and soft tissues in PC and DC images of the entire adult human hand, and to provide a potential application tool for integrating this new information into conventional radiography.

\section{Materials and methods}

Human cadaver hand specimen

A total of three human cadaver hands of a 54-, a 65-, and a 73-year-old man (cause of death unknown) were resected $3 \mathrm{~cm}$ proximal to the radiocarpal joint. Prior to imaging, the hand was perfused with Thiel solution according to standard procedures to preserve soft tissue integrity and architecture [14]. Cadaver hands were used in accordance with institutional ethical laws and regulations after prior approval by the department of anatomy.

\section{Imaging setup}

A schematic drawing of the technical setup is shown in Fig. 1a. Along with an X-ray source (Seifert ID 3000 tube) and a pixel detector (Hamamatsu flat-panel C9732DK, 50× $50 \mu \mathrm{m}$ pixel size), which are standard components in conventional radiography, three optical devices, referred to as gratings, are arranged at predefined positions between the source and the detector. The structures of the gratings resemble lamellae (Fig. 1b), having periodicities in the order of micrometers. Their fabrication requires advanced lithographic processes $[15,16]$. The arrangement of the three gratings is called a grating interferometer; the technique itself is referred to as X-ray grating interferometry. More details about the technique and the retrieval of absorption, PC and DC images are given in the Supplemental materials section. The technical specifications of the grating interferometer setup used in this experiment are listed in Table 1. The X-ray source was operated at an acceleration voltage of $40 \mathrm{kV}$ and a current of $25 \mathrm{~mA}$ and the design energy of the grating interferometer was $28 \mathrm{keV}$.

\section{Data acquisition}

Because of the limitation of the field of view by the size of the gratings and the geometric magnification of the sample to an area of $50 \times 50 \mathrm{~mm}^{2}$, a mesh scan $(4 \times 5 \mathrm{mesh})$ had to be performed to cover the entire sample.

Other than the conventional absorption image, the PC and DC images are sensitive to object orientation. Refraction or scattering of X-rays can occur in all directions in the image plane, whereas the grating interferometer is only sensitive in one direction (i.e., in the direction perpendicular to the grating lines). If there is, for instance, an interface of two materials that is horizontally oriented, refraction occurs only in the vertical direction. This interface will not be recorded if the grating interferometer is sensitive in the horizontal direction only (i.e., with vertical grating lines). Therefore, a bidirectional scanning approach was used, which solves this limitation by acquiring two scans where the sample for the second scan is rotated by $90^{\circ}$ with respect to the first scan [12]. In order to precisely rotate the sample by $90^{\circ}$ after the first scan, the samples were fixed on a ground plate, which was mounted on a rotating axis around the z-axis. Alternatively, bi-directional sensitivity could also be obtained without rotating the sample by using two-dimensional checkerboard (G1) and mesh gratings [17].

Post-processing and image fusion

In a first step of this study, the raw images of each contrast mode, namely the absorption, PC, and DC images, have been evaluated as gray scale images. Since the bi-directional scanning approach yields two images, which differ in the PC and 
a

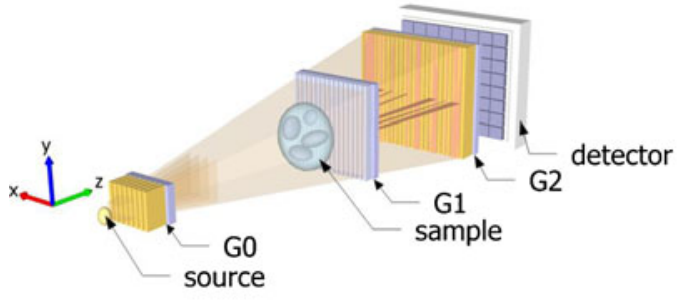

b

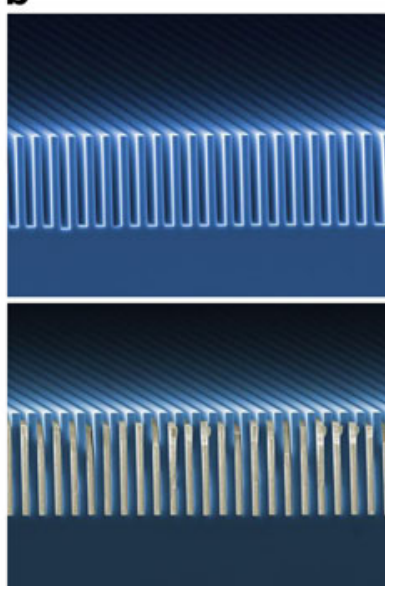

Fig. 1 a Schematic drawing of a grating interferometer setup. The Xray source depicts the point where the accelerated electrons hit the target in the tube. Immediately after the source, the source grating $(G 0)$ is placed, splitting the beam into an array of small sources, which is necessary for the interference formation. Further downstream, the beam splitter grating $(G 1)$ generates a periodic interference pattern

the DC modes, they have been processed into a single image by using the following operations:

$$
\begin{gathered}
P_{i}=\sqrt{P_{x, i}^{2}+P_{y, i}^{2}} \\
D_{i}=\frac{1}{2}\left(D_{x, i}+D_{y, i}\right)
\end{gathered}
$$

$P_{\mathrm{x}}$ and $D_{\mathrm{x}}$ are the $\mathrm{PC}$ and DC images respectively from the first scan, whereas $P_{\mathrm{y}}$ and $D_{\mathrm{y}}$ are the images obtained from the second scan with the sample rotated by $90^{\circ}$. The index $i$ represents the pixel index and indicates that image processing is performed independently in each pixel.

In a second step, the PC image was overlaid by the absorption or the DC image to simultaneously display the different contrast modalities. Details of the image fusion method used are discussed in section $\mathrm{S} 2$ of the supplemental material. While DC and absorption images reveal higher contrast for bones,

Table 1 Parameters of the setup geometry and the gratings

\begin{tabular}{ll}
\hline Parameter & Value \\
\hline Design energy & $28 \mathrm{keV}$ \\
Distance source-to-G0 & $2 \mathrm{~mm}$ \\
Distance G0-to-G1 & $1,400 \mathrm{~mm}$ \\
Distance G1-to-G2 & $200 \mathrm{~mm}$ \\
Distance G2-to-detector & $10 \mathrm{~mm}$ \\
G0 pitch & $14.2 \mu \mathrm{m}$ \\
G0 height & $50 \mu \mathrm{m}$ \\
G1 pitch & $3.51 \mu \mathrm{m}$ \\
G1 height & $36 \mu \mathrm{m}$ \\
G2 pitch & $2.0 \mu \mathrm{m}$ \\
G2 height & $25 \mu \mathrm{m}$ \\
G2 active area & $64 \times 64 \mathrm{~mm}^{2}$ \\
\hline
\end{tabular}

(fringes) with a maximum intensity modulation at $G 2$. G2 itself is the analyzer grating, used to sense changes in the interference fringes, which were caused by attenuation, refraction or scattering of the Xrays by the sample. b Scanning electron microscope images of the grating structures of a phase grating made with silicon lines (top) and an absorption grating of gold lines (bottom)

and the PC image mainly contains soft tissue features, the fusion of the PC image with either of the two other contrasts includes both. By further color coding the PC image, the superimposed images can still be easily distinguished. A main feature of this type of fusion is the control of the opacity of the PC image, which can be continuously adjusted by the user.

\section{Image interpretation}

Two experienced radiologists (with 5 and 10 years of experience) who were blinded to each other subjectively evaluated visualization of subchondral bone (depiction quality of microtrabeculae, subchondral cysts and erosions if present), joint space (including periarticular calcifications if present), and soft tissues (depiction quality of soft tissue septae) on absorption plain radiographs, differential PC and DC images (Fig. 2). A four-point Likert scale was used $(0=$ not visible or bad visualization, $1=$ probably visible with moderate visualization, $2=$ visible with good visualization, $3=$ clearly visible with perfect visualization). It was developed as a trade-off between the best characteristics of different joint compartments using a single graduation scale on the one hand and established scoring methods for plain radiographs on the other hand. The scale was therefore based on the widely used Sharp/van der Heijde and Kellgren/Lawrence scales [18, 19] for the quantification of bone erosions, osteophytes, sclerosis, joint space narrowing, and joint subluxation, but adapted to a mere visualization aspect of joint components, i.e., subchondral bone, joint space, and soft tissues. The three joint components were evaluated for the radiocarpal, carpal, metacarpo-phalangeal, proximal interphalangeal, and distal interphalangeal joint groups of 

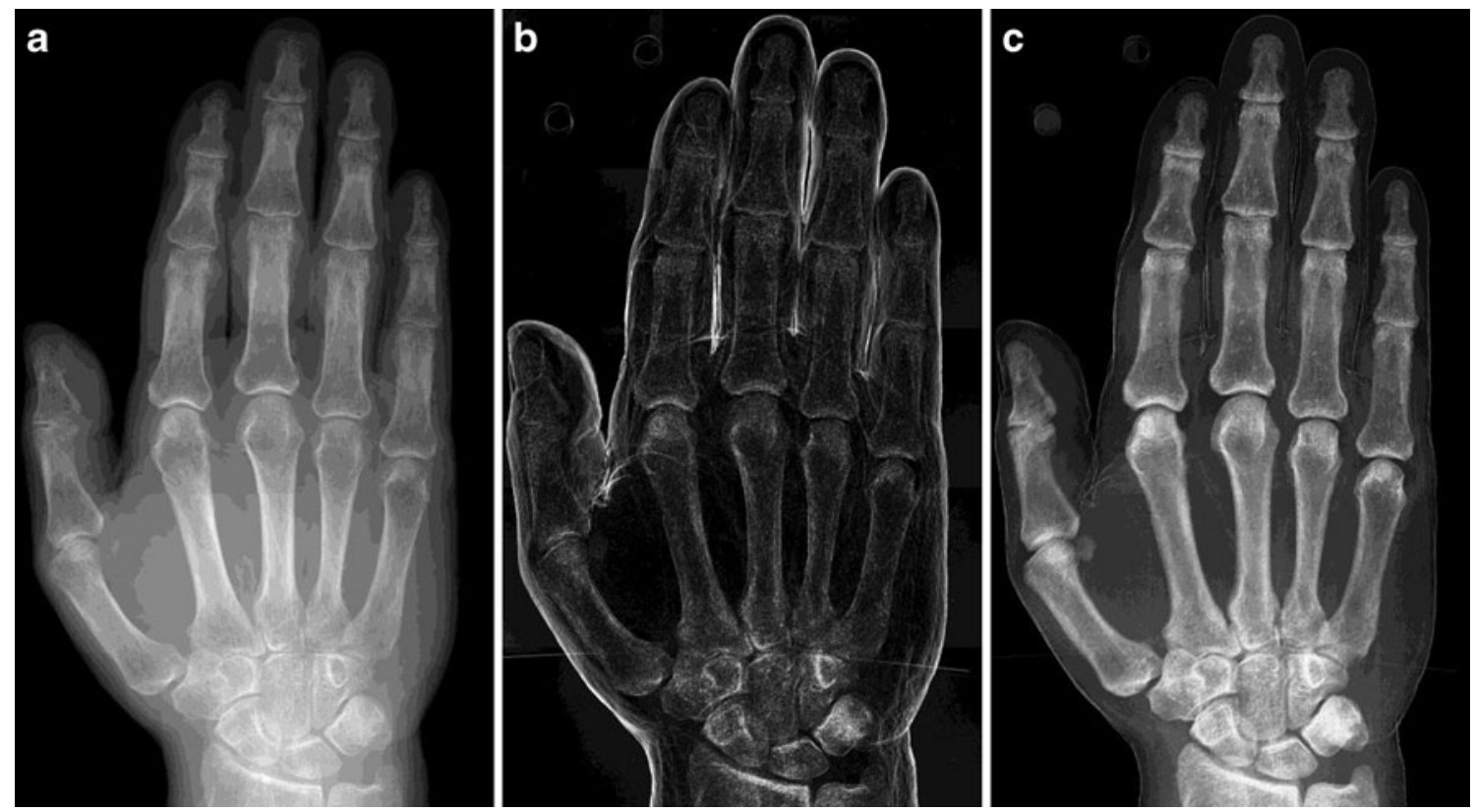

Fig. 2 Images of a human cadaver hand in the three contrast modes, acquired with the grating interferometer: a standard absorption contrast, b phase contrast (PC), and $\mathbf{c}$ dark-field contrast

each hand. Eventually, fused PC/absorption and fused $\mathrm{PC} / \mathrm{DC}$ images were evaluated in consensus by both readers at different opacity levels of the PC image, and the visualization of joint structures was assessed.

\section{Statistical analysis}

Data analysis was performed using commercially available software (IBM SPSS Statistics Version 20, release 20.0.0, Chicago, IL, USA). Statistical significance was inferred at a $p$ value below 0.05 . Continuous variables are expressed as mean \pm standard deviation.

Inter-reader agreement was determined by weighted kappa statistics. A kappa value from 0.41 to 0.60 was interpreted as moderate, $0.61-0.80$ as substantial, and $0.81-1$ as almost perfect agreement. Differences in ratings between images were assessed using paired Wilcoxon signed-rank tests with Bonferroni corrections for multiple comparisons, using a corrected $p$ value of $<0.017$ to indicate statistical significance.

\section{Results}

Inter-reader agreement

Descriptive data (i.e., mean and median ratings with standard deviations, kappa and $p$ values) for both readers, image types, and joint structures are listed in Table 2. Inter-reader agreement for visualization was moderate for all structures
(DC) images. The PC image encodes the refraction of the X-rays inside the sample, the DC image the scattering intensity, into gray value images

in conventional radiography and for soft tissue in DC images ranging between 0.42 and $0.44(p=0.057-0.13)$, while being substantial and significant for all structures on PC, and for subchondral bone and joint space on DC images $(0.70-0.88, p<0.05)$.

Image quality and imaging findings

Significant differences in visualization ratings were found between the various image types. Soft tissue visualization was rated higher on PC compared with both absorption and DC images $(p<0.017)$, whereas the subchondral bone and joint space could be better assessed on absorption and DC images $(p<0.001)$. Joint space visualization was rated significantly higher on DC images than on conventional radiography $(p<0.017)$, while visualization of other structures did not differ significantly.

The absorption, PC, and DC images of the human hand were separately depicted as gray value images in Fig. 2. The difference and the complementary information content can already be identified from the unprocessed (or unfused), raw contrast modalities.

Both radiologists found the gray value PC images to generally enhance contrast for soft tissue. The DC image, on the other hand, shows enhanced contrast of bone edges versus soft tissue with better visualization of the joint space and bone margins compared with conventional radiography.

On conventional radiography, one of the cadaver hands revealed coarse calcifications in the triangulo-fibrocartilage 
Table 2 Image quality ratings of both readers for all image types

\begin{tabular}{|c|c|c|c|c|c|c|c|c|c|}
\hline \multirow[t]{2}{*}{ Image type } & \multirow[t]{2}{*}{ Structure } & \multicolumn{3}{|c|}{ Reader 1} & \multicolumn{3}{|c|}{ Reader 2} & \multirow[t]{2}{*}{ kappa } & \multirow[t]{2}{*}{$p$ value } \\
\hline & & Mean & Median & $\pm \mathrm{SD}$ & Mean & Median & $\pm \mathrm{SD}$ & & \\
\hline \multirow[t]{3}{*}{ Absorption } & Subchondral bone & 2.2 & 2 & 0.44 & 2.3 & 2 & 0.48 & 0.42 & 0.13 \\
\hline & Joint space & 2.1 & 2 & 0.28 & 2.2 & 2 & 0.44 & 0.44 & 0.057 \\
\hline & Soft tissue & 1.9 & 2 & 0.28 & 1.8 & 2 & 0.44 & 0.44 & 0.057 \\
\hline \multirow[t]{3}{*}{ PC } & Subchondral bone & 0.2 & 0 & 0.44 & 0.3 & 0 & 0.48 & 0.81 & $<0.05$ \\
\hline & Joint space & 0.5 & 0 & 0.66 & 0.4 & 0 & 0.51 & 0.71 & $<0.05$ \\
\hline & Soft tissue & 2.3 & 2 & 0.48 & 2.3 & 2 & 0.63 & 0.70 & $<0.05$ \\
\hline \multirow[t]{3}{*}{$\mathrm{DC}$} & Subchondral bone & 2.3 & 2 & 0.75 & 2.2 & 2 & 0.73 & 0.88 & $<0.001$ \\
\hline & Joint space & 1.7 & 2 & 0.75 & 1.7 & 2 & 0.75 & 0.72 & $<0.001$ \\
\hline & Soft tissue & 1.7 & 2 & 0.48 & 1.8 & 2 & 0.44 & 0.42 & 0.125 \\
\hline
\end{tabular}

$P C$ phase contrast image, $D C$ dark-field contrast image, $\pm S D \pm$ standard deviation

complex of the radiocarpal joint. Visualization of this finding was markedly increased on DC images (Fig. 3). Another calcification adjacent to the distal interphalangeal joint could be only depicted on DC images, but not on conventional radiography and $\mathrm{PC}$ images (Fig. 4).

Fused images

For the fusion of the PC image with the absorption or the DC image respectively, the method described in S2 of the supplemental material has been used. Fig. 5 shows the fusion of the PC image with the absorption image and Fig. 6 the fusion of the PC and the DC image. The opacity of the PC image, coded in blue, can be continuously adjusted by a weighting parameter $\alpha_{A}$ (PC/absorption) and $\alpha_{D}$ (PC/DC). In order to emphasize the practical meaning of the weighting parameters $\alpha_{A}$ and $\alpha_{D}$, both figures show three different images with a varying $\alpha$.

Both radiologists found the fused absorption and PC images to yield higher contrast between soft tissue and bones compared with standard absorption radiographs. The visibility of soft-tissue features was enhanced by increasing the weighting parameter, yet slightly more obscuring the bony microarchitecture.

Tissue contrast between soft tissue and bones was further enhanced by the fusion of PC and DC images. As already seen on raw images, DC images markedly enhanced the visualization of bones. Increasing the weighting factor of the fused images increased soft tissue contrast and simultaneously maintained the visibility of bony microarchitecture on the DC image.

\section{Dose}

The mean dose rate within the field of view was measured to be $0.7 \mathrm{mGy} / \mathrm{s}$. By using eight phase steps [20] and an exposure time of $9 \mathrm{~s}$ per step, the total entrance dose delivered amounts to $0.7 \mathrm{mGy} / \mathrm{s} \cdot 8 \cdot 9 \mathrm{~s} \cdot 2=100.8 \mathrm{mGy}$. The factor of 2 takes into account the bi-directional measurement. Alternatively, uni-directional measurements could have been used to reduce the dose; however, the sensitivity
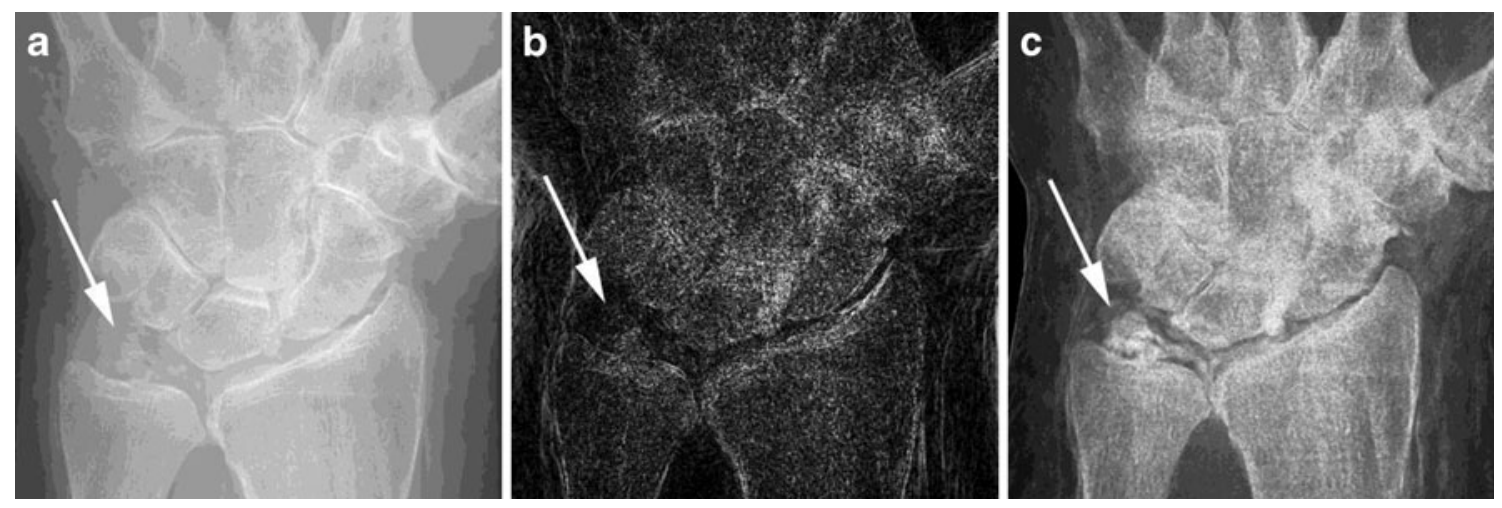

Fig. 3 a On the absorption radiograph image, one of the hands revealed calcifications in the triangulo-fibrocartilage complex (TFCC) of the radiocarpal joint (arrow). b Soft tissue was better depicted on the
PC image; however, differentiation of TFCC calcifications (arrow) was less clear. c Visualization of TFCC calcifications (arrow) was best on DC images 

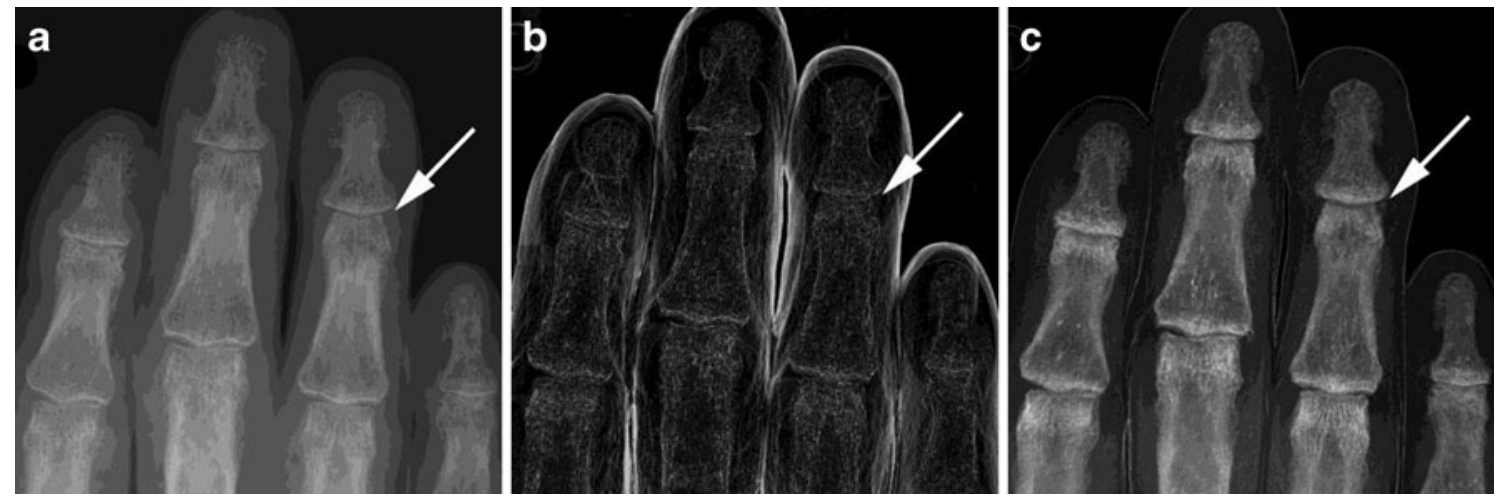

Fig. 4 Soft tissue calcification adjacent to the distal interphalangeal joint of the fourth finger (arrows) was not depicted on a the absorption radiograph or on $\mathbf{b}$ the $\mathrm{PC}$ image, but could be clearly seen on $\mathbf{c}$ the DC image

of the PC and DC images would then be reduced to one direction.

The relatively high dose rate can partly be explained by the fact that the system has not been optimized for this purpose. After the sample, two Plexiglas plates, used to protect the interferometer, attenuate the radiation by approximately $40 \%$, which represents a post-sample attenuation factor and reduces the detection efficiency. Furthermore, the $\mathrm{X}$-ray detector is optimized for $17 \mathrm{keV}$, while the interferometer's design energy is $28 \mathrm{keV}$. The corresponding loss in detection efficiency has been estimated to be about 4-fold. Also, the spectrum has not been optimized, e.g., by using filters. By optimizing the setup and thus the associated detection efficiency (see above), a dose reduction by a factor of approximately 8 could be achieved, which would result in an entrance dose of $12.6 \mathrm{mGy}$ for a bi-directional measurement or $6.3 \mathrm{mGy}$ for a uni-directional measurement.

\section{Discussion}

The experiments presented here show a study on PC and DC imaging in a large field of view for adult human hands and demonstrate the potential of this technique to improve diagnosis. It was found that PC images significantly improve the visualization of soft tissue of the hand (i.e., by highlighting
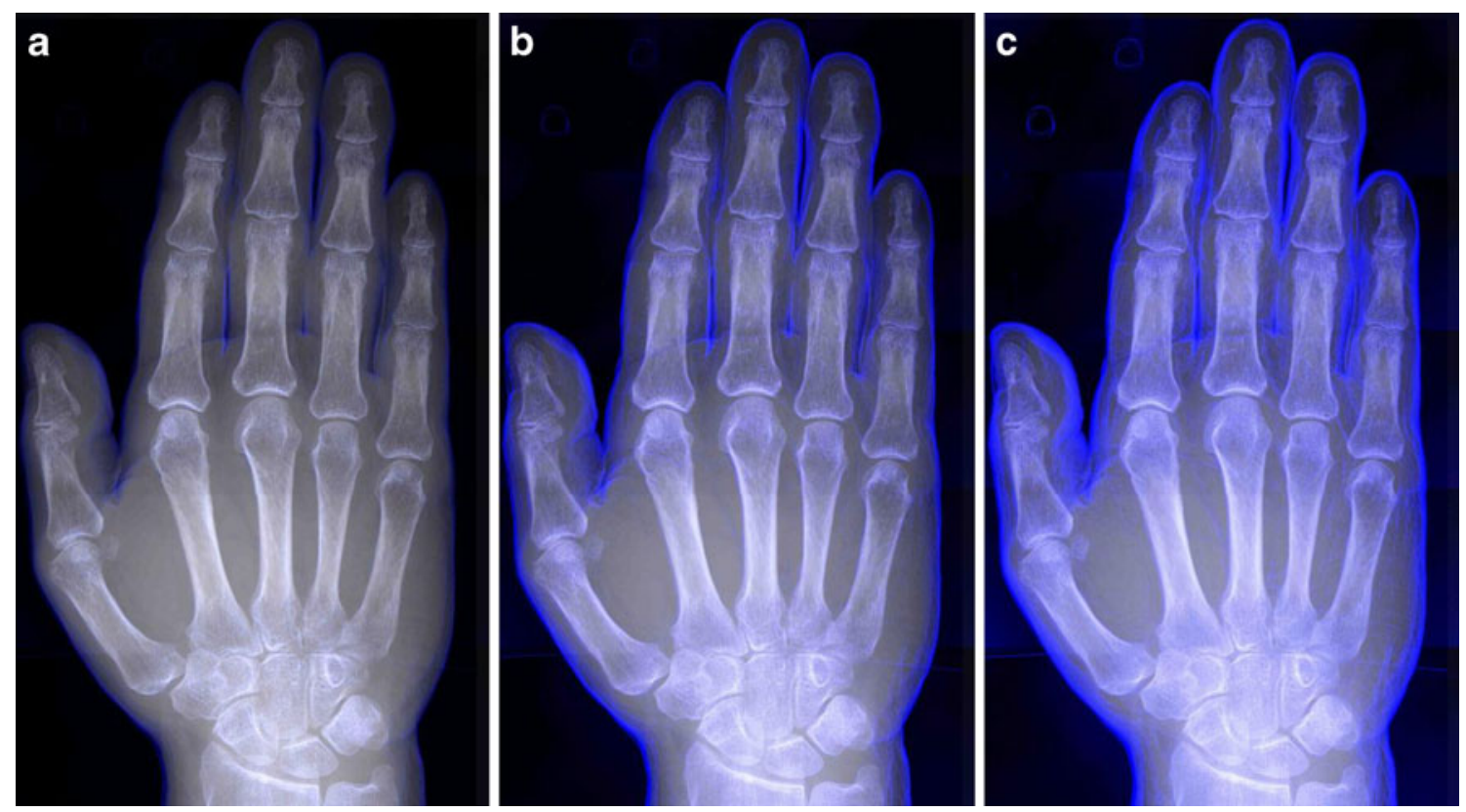

Fig. 5 Fusion of the standard absorption radiograph with the PC image, using different values for the fusion parameter alpha (see S2 of the supplemental material). a $\alpha_{\mathrm{A}}=1, \mathbf{b} \alpha_{\mathrm{A}}=3, \mathbf{c} \alpha_{\mathrm{A}}=6$. A small value for the fusion parameter results in an image that is dominated by the absorption image, any soft tissue features provided by the PC image are invisible. By increasing the fusion parameter, the visibility of those soft tissue features can be continuously enhanced 

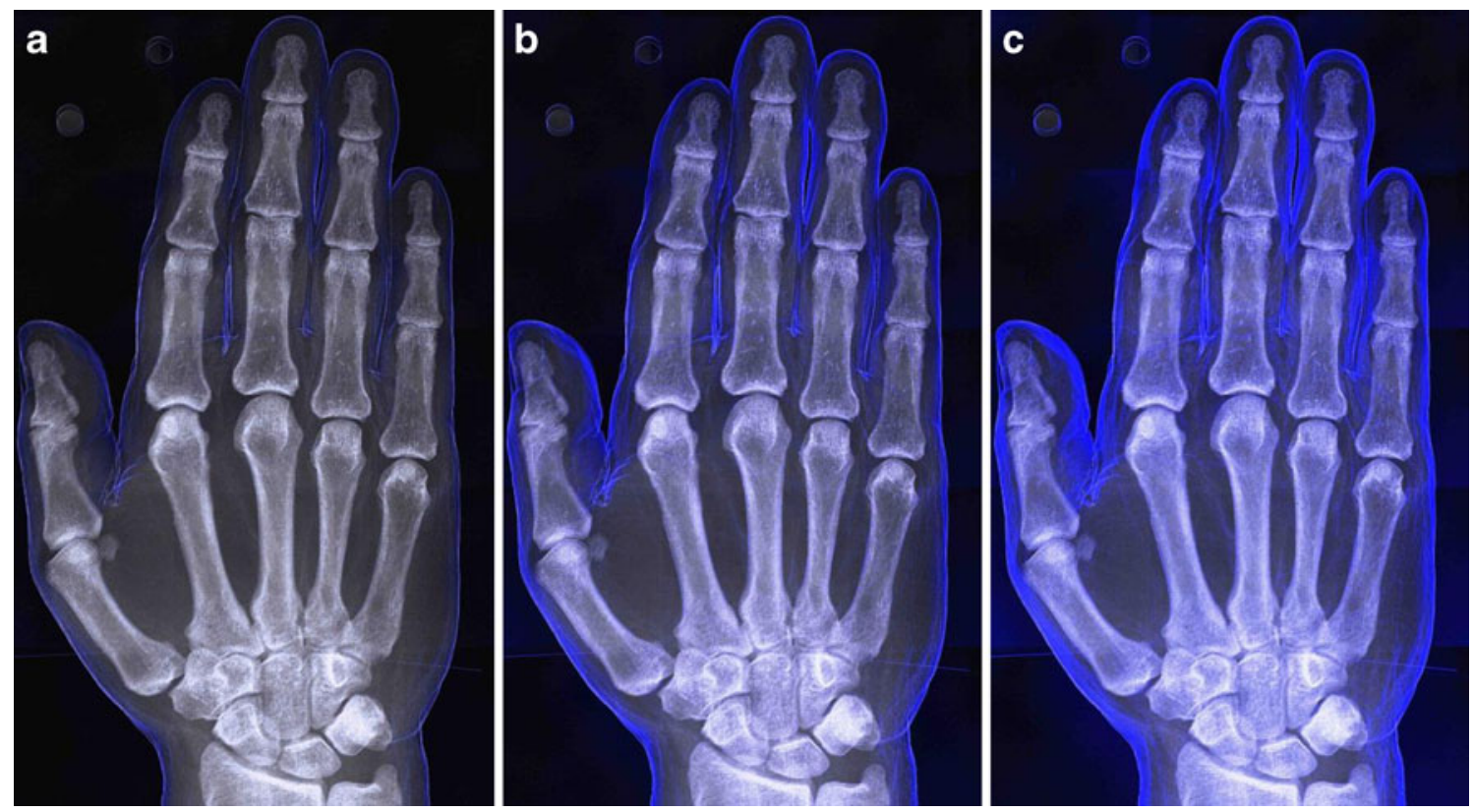

Fig. 6 Fusion of the mean scattering signal with the DC image, using different values for the fusion parameter alpha (see S2 of the supplemental material). a $\alpha_{\mathrm{D}}=1, \mathbf{b} \alpha_{\mathrm{D}}=3$, c $\alpha_{\mathrm{D}}=6$. Again, a higher fusion

fibrous septa and interfaces), whereas DC images improve the image quality of bony edges and margins leading to increased visualization of bone microarchitecture and joint spaces with periarticular calcifications. Image fusion has been used for the simultaneous display of the different image types, where the opacity of the overlaid color-coded PC image onto the absorption or DC image could be changed manually and interactively by the readers from none to maximal according to individual preferences. Both radiologists found the fused absorption and PC images to yield higher contrast between soft tissue and bones compared with conventional radiography. Further contrast increase of bone margins and microarchitecture was achieved by the fusion of DC images with PC images. Although this is an experimental study using a highly dedicated setup and cadaver hands, the image fusion method used has, because of its interactive functionality of continuously changing the opacity of the PC image, the potential to enter clinical utility in daily routine, possibly finding a role in diagnosing and monitoring rheumatological and degenerative disease.

For decades, conventional radiography has been the cornerstone imaging examination for the diagnosis and monitoring of joint disease, such as osteoarthritis or rheumatoid arthritis, and remains a valuable tool at present owing to the high resolution depiction of joint structures and associated diseases [21, 22]. However, in recent years magnetic resonance (MR) imaging and ultrasound have attracted increasing interest for monitoring disease evolution because of their potential to detect soft-tissue affection, such as synovial thickening, perfusion or joint effusions at an earlier stage parameter leads to greater visibility of the PC image and, therefore, of soft tissue features

than plain radiography. Guidelines on disease classification and treatment strategies have therefore integrated findings from MR and ultrasound imaging in order to facilitate earlier initiation or changes in therapeutic regimen [23]. However, MR imaging is rather expensive, more limited in resolution, and not always available. Although ultrasound imaging has been widely introduced into the rheumatological community, it is rather time consuming and its performance depends on the user's expertise. Plain radiography on the other hand can be performed quickly at low costs with constantly high quality, leaving little space for variance of acquisition quality, although at the price of an ionizing radiation dose.

Various attempts have been made to quantify joint disease by using standardized read-out schemes for conventional radiography; however, currently, there is no consensus on the preferred scale [19, 24, 25]. Although controversially discussed, the Kellgren and Lawrence approach [19] is widely used as a tool for systematic reports of hand conventional radiography. This algorithm is based on the assessment of typically affected joint structures in osteoarthritis, such as subchondral bone and joint space width. Therefore, the visualization of subchondral bone, joint space, and, in addition, soft tissue structures in different joints of the hand to investigate the potential value of the various image types for joint disease assessment, was evaluated.

Grating interferometry-based PC and DC imaging must be considered one of the most promising phase contrast techniques in terms of clinical application, since it can be 
technically realized with conventional X-ray tubes. The source-to-detector length of $1.6 \mathrm{~m}$ in the system used for our study was rather long; however, it has been demonstrated that the technique is also compatible with more compact settings [26]. The geometry of the grating interferometer is usually fixed for a given design energy, which makes it less flexible than a standard absorption setup. Compared with the standard acquisition protocol in absorption imaging, which is based on a single snap of the sample, grating interferometry requires a multi-snap acquisition protocol of typically 4-8 images. This can increase scan time because of the idle time between the exposures; however, the dose can be kept constant by reducing the exposure time of the single snaps. In a clinical context, the increased scan time might become a problem owing to the occurrence of patient motion and the associated image blurring. Another issue related to blurring is the intrinsic geometric magnification of phase contrast images. Since the sample is placed in front of the phase grating (middle grating), there is always a certain distance between the sample and the detector. The finite focal spot size of the source can cause a geometric unsharpness (penumbra), which appears as blurring on the images. An absorption image acquired with a standard setup would of course not be affected by such blurring. On the other hand, this effect can be avoided by selecting an X-ray tube with a smaller focal spot, allowing the required geometric magnification.

The following study limitations must be acknowledged. First, the ex vivo approach has inherent shortcomings. Second, there is no gold standard modality available for correlating findings from PC and DC images. Third, only three hands were included and evaluation of depiction quality did not focus on specific findings of a certain pathology, i.e., rheumatoid arthritis. Performance of PC and DC images in clinical situations will have to be tested in further studies. Finally, the experimental set-up is not yet ready for clinical use. On the one hand, the field of view of a single exposure is too small to cover the entire human hand; therefore, multiple exposures, ideally with some overlapping space to simplify the subsequent stitching of the scans, have to be acquired. On the other hand, the system has not been optimized for the minimization of the dose imparted.

Phase contrast and dark-field contrast imaging of the human adult hand using a conventional X-ray tube are feasible and provide advantages in the depiction of both bone and soft tissue compared with conventional radiography. Use of a fusion tool has the potential to highlight the individual value of each image type, deserving further evaluation. Finally, early evidence suggests that soft tissue calcifications can be depicted with better quality on DC compared with conventional radiography images. Thus, once established in clinical routine, $\mathrm{PC}$ and DC imaging may hold potential as imaging tools for the more accurate diagnosis and monitoring of rheumatological disease.
Acknowledgements The authors thank Gordan Mikuljan from Paul Scherrer Institute for the design and manufacturing of various mechanical components. They also thank Prof. Verdun from the Institute of Radiation Physics at the University Hospital center Lausanne for his competent help in dose calculations. Part of this work has been supported by the ERC Grant ERC-2012-StG 310005-PhaseX.

\section{References}

1. David C, Nöhammer B, Solak H, Ziegler E. Differential x-ray phase contrast imaging using a shearing interferometer. Appl Phys Lett. 2002;81(17):3287-9.

2. Pfeiffer F, Weitkamp T, Bunk O, David C. Phase retrieval and differential phase-contrast imaging with low-brilliance $\mathrm{X}$-ray sources. Nat Phys. 2006;2(4):258-61.

3. Engelhardt M, Baumann J, Schuster M, Kottler C, Pfeiffer F, Bunk $\mathrm{O}$, et al. High-resolution differential phase contrast imaging using a magnifying projection geometry with a microfocus $\mathrm{x}$-ray source. Appl Phys Lett. 2007;90(22):224101.

4. Pfeiffer F, Bech M, Bunk O, Kraft P, Eikenberry E, Brönnimann C, et al. Hard-X-ray dark-field imaging using a grating interferometer. Nat Mater. 2008;7(2):134-7.

5. Engel KKJK, Geller D, Köhler T, Martens G, Schusser S, Vogtmeier $\mathrm{G}$, et al. Contrast-to-noise in X-ray differential phase contrast imaging. Nucl Inst Methods Phys Res A. 2010;648:202-7.

6. Stampanoni M, Wang Z, Thüring T, David C, Roessl E, Trippel M, et al. The first analysis and clinical evaluation of native breast tissue using differential phase-contrast mammography. Investig Radiol. 2011;46(12):801-6.

7. Steinbach L, Resnick D. Calcium pyrophosphate dihydrate crystal deposition disease: imaging perspectives. Curr Probl Diagn Radiol. 2000;29(6):209-29.

8. Koutaissoff S, Vanthuyne M, Smith V, De Langhe E, Depresseux G, Westhovens R, et al. Hand radiological damage in systemic sclerosis: comparison with a control group and clinical and functional correlations. Semin Arthritis Rheum. 2011;40(5):455-60.

9. Coan P, Bamberg F, Diemoz P, Bravin A, Timpert K, Mützel E, et al. Characterization of osteoarthritic and normal human patella cartilage by computed tomography X-ray phase-contrast imaging: a feasibility study. Investig Radiol. 2010;45(7):437-44.

10. Stutman D, Beck TJ, Carrino JA, Bingham CO. Talbot phasecontrast $\mathrm{x}$-ray imaging for the small joints of the hand. Phys Med Biol. 2011;56(17):5697-720.

11. Donath T, Pfeiffer F, Bunk O, Grünzweig C, Hempel E, Popescu S, et al. Toward clinical X-ray phase-contrast CT: demonstration of enhanced soft-tissue contrast in human specimen. Investig Radiol. 2010;45(7):445-52.

12. Jensen T, Bech M, Zanette I, Weitkamp T, David C, Deyhle H, et al. Directional x-ray dark-field imaging of strongly ordered systems. Phys Rev B. 2010;82(21):214103.

13. Kottler C, David C, Pfeiffer F, Bunk O. A two-directional approach for grating based differential phase contrast imaging using hard x-rays. Opt Express. 2007;15(3):1175-81.

14. Thiel W. Die Konservierung ganzer Leichen in natürlichen Farben. Ann Anat Anat Anzeiger. 1992;174(3):185-95.

15. David C, Bruder J, Rohbeck T, Grünzweig C, Kottler C, Diaz A, Fabrication of diffraction gratings for hard X-ray phase contrast imaging. Microelectron Eng. 2007;84(5-8):1172-1177.

16. Kenntner J, Grund T, Matthis B, Boerner M, Mohr J, Scherer T et al. Front- and backside structuring of gratings for phase contrast imaging with x-ray tubes. Proc SPIE. 2010. 10.1117/12.859666 
17. Zanette I, Weitkamp T, Donath T, Rutishauser S, David C. Twodimensional X-Ray grating interferometer. Phys Rev Lett. 2010;105:248102.

18. Van der Heide D. How to read radiographs according to the Sharp/ van der Heijde method. J Rheumatol. 2000;27(1):261-3.

19. Kellgren J, Lawrence J. Radiological assessment of osteoarthrosis. Ann Rheum Dis. 1957;16(4):494-502.

20. Weitkamp T, Diaz A, David C, Pfeiffer F, Stampanoni M, Cloetens P, et al. X-ray phase imaging with a grating interferometer. Opt Express. 2005;12(16):6296-304.

21. Fouque-Aubert A, Boutroy S, Marotte H, Vilayphiou N, Lespessailles E, Benhamou C, et al. Assessment of hand trabecular bone texture with high resolution direct digital radiograph in rheumatoid arthritis: a case control study. Joint Bone Spine. 2012;79(4):379-83.
22. Pye S, Adams J, Ward K, Brunn D, Symmons D, O'Neill T. Disease activity and severity in early inflammatory arthritis predict hand cortical bone loss. Rheumatology. 2010;49(10):1943-8.

23. Aletaha D, Neogi T, Silman A, Funovits J, Felson D, Bingham C, et al. 2010 Rheumatoid arthritis classification criteria: an American College of Rheumatology/European League against Rheumatism collaborative initiative. Arthritis Rheum. 2010;62(9):2569-81.

24. Spector T, Cooper C. Radiographic assessment of osteoarthritis in population studies: whither Kellgren and Lawrence? Osteoarthr Cartil. 1993;1:203-6.

25. Altman RD, Gold GE. Atlas of individual radiographic features in osteoarthritis, revised. Osteoarthr Cartil. 2007;15:A1-A56.

26. Thuering T, Modregger P, Grund T, Kenntner J, David C, Stampanoni M. High resolution, large field of view x-ray differential phase contrast imaging on a compact setup. Appl Phys Lett. 2011;99(4):041111. 